\title{
El origen de la clínica del dolor
}

\author{
Darío Zuleta Olano*
}

El tratar de explicar el origen de las clínicas del dolor, nos lleva necesariamente a incursionar sobre la existencia del hombre, su proceso social e histórico. Es muy probable que por este camino se dé razón clara del porqué del concepto moderno para el alivio del hombre adolorido.

De pronto no es muy significativo señalar cronológicamente los diferentes hechos que han marcado épocas en el desarrollo científico-técnico, pero sí anotar que como consecuencia de los avances logrados por la ciencia y la técnica, se va logrando la tendencia cada día mayor, a articular una ciencia en torno a la complejidad del ser humano y su relación con la naturaleza: "La ciencia del Hombre".

Vale la pena entonces anotar que la tendencia antigua de considerar el estudio del hombre con base a sus cualidades material-estructural (anatómicas únicamente) dio paso a una segunda época, que se caracterizó por la explicación funcional del hombre (fisiología-funcionalismo).

Al dejar espacios en blanco estas dos teorías en el conocimiento del problema del hombre, se llega al enfoque holístico y la concepción sistémica de él, al considerarlo como un sistema, que pertenece a su vez a un sistema mayor, pasando entonces del enfoque biologista dado por las dos primeras épocas de desarrollo, a considerar el objeto de estudio (en este caso el hombre) en toda su dimensión de ser humano, con aptitudes y personalidad incluidas (Ser sicológico). Pero también como individuo social, vinculado e interactuando con el grupo social del cual forma parte (ser genérico-cultural).

Es válido en este momento aclarar que si la tendencia actual de la ciencia apunta hacia la integración de los diferentes campos de la ciencia con miras a ir obteniendo la percepción objetiva del mundo, también es válido aceptar que aún en nuestros días el que hacer general está regido por el abordaje principalmente biologista (material-estructural y/o funcional), a manera de ilustrar introduzco algunos conceptos que son cotidianos en las Instituciones hospitalarias: "El Infarto Masivo de la cama 301", "La Coledocolitiasis de la 604" "La Eclampsia de la 202", "La sicosis maníacodepresiva de la 115". Estos ejemplos para situarnos únicamente en el campo de las ciencias de la salud.

El abordaje original propuesto para la clínica del dolor es trascender más allá de lo puntual biológico, lo fríamente técnico, para retomar la categoría de lo humano como esencial. En este marco teórico holístico funciona nuestra idea de clínica del dolor.

* Profesor Asociado. Departamento de Rehabilitación. Facultad de Medicina. Universidad Nacional de Colombia.
Una cualidad ya observada, con la idea de clínica del dolor, ha sido la de aceptar la complejidad del ser humano que se queja de dolor. De lo anterior se ha desprendido la necesidad de imponer el abordaje del objeto de estudio, con la participación de profesionales de diferentes disciplinas de ciencias de la salud, con interacción, de manera coordinada.

Un punto de reflexión es necesario hacer en este momento y es que la mayoría de clínicas de dolor, aún no se han salido del enfoque puramente curativo. Es una tarea que en nuestro caso va tomando mayor espacio, el de establecer medidas preventivas que controlen la aparición del dolor, en cuanto sean prevenibles las causas que lo generen.

Son muchos los esfuerzos que se han realizado tratando de alcanzar la atención lo más humana y científica del hombre. En el campo de la salud y en lo referente al tema de la atención del hombre con dolor, vale la pena destacar a nivel mundial entre otros a: Dr. John J. Bonica, R.G. Twy Cross, R. Melzack, Dr. Patric Wall, Dra. K. Foley, Dra. C.M. Saunders. A nivel latinoamericano: Drs. Fiscela y Cerezo de Argentina, Ramón de Tille de Méjico, Bento Gonzalves de Brasil, en Colombia, los anestesiólogos Drs. Tiberio Alvarez y Pedro Bejarano.

Con la idea de contribuir al conocimiento del hombre y con la intención de no perpetuar el abordaje simplista de la realidad hombre-dolor y por el contrario tratando de conseguir hacerlo de manera cabal, en el año de 1987, presenté ante un grupo de profesionales de la salud, algunos de ellos vinculados con la Universidad Nacional como docentes, pero que laboraban en una de las cuatro Instituciones que se han llamado a conformar el "Centro Médico Nacional" y que son: Hospital San Juan de Dios, Instituto Materno Infantil, Instituto de Cancerología y Hospital de la Misericordia, el proyecto de creación de "clínica para estudio, manejo y alivio del paciente con dolor", que además de conjugar al conocimiento de varias disciplinas científicas, fuera interinstitucional, ya que los nexos de territorialidad lo facilitaban. Fue así como recibidas las sugerencias de algunos de dichos profesionales, entre quienes puedo mencionar a los profesores Fernando Flórez, Juan Trujillo M., Héctor Tejada H., se conformó el proyecto logrando su oficialización a nivel de la Facultad de Medicina de la Universidad Nacional y las Instituciones asistenciales antes mencionadas. El progreso en el estudio, el aumento en la demanda de servicios y la inquietud por el enfoque dado, ha venido incluyendo profesionales destacados en diferentes campos de ciencias de la salud como son: el Dr. Pío Iván Gómez y el Dr. Antonio Medina en el Instituto Materno Infantil, la Dra. Adelaida Martínez y el Dr. Rafael Vásquez 
en el Hospital de la Misericordia, y John Hernández en el Hospital San Juan de Dios. Vale la pena destacar que en la primera etapa de constitución fueron pilares permanentes la Sicóloga Dra. Fany de Laverde, la Trabajadora Social Patricia Duque, del Hospital San Juan de Dios. En el Instituto de Cancerología el Dr. Daniel Gutiérrez, la Trabajadora Social María Eugenia Saiz M. y la Sicóloga Vivian Perilla.

El gran desarrollo de la clínica llevó en una etapa posterior a la creación de coordinadores en cada una de las Instituciones antes enunciadas y a la vinculación de otros valiosos profesionales que vienen prestando sus servicios con gran sentido humanitario.

Es de justicia mencionar aquí el trabajo permanente y con gran entrega que han venido brindando los estudiantes de postgrado del Departamento de Rehabilitación de la Universidad Nacional y algunos del Instituto de Cancerología.

\section{El quehacer de la clínica del dolor}

La actividad cotidiana de la clínica del dolor puede concretarse en tres direcciones:
- De atención a la persona: con la modalidad de consulta interdisciplinaria (sicólogo, trabajador social, enfermera, médicos de diferentes especialidades) se dialoga con el paciente y sus familiares. El análisis cabal de la situación de cada persona conduce a la toma de decisiones integradas, tomando a la persona en problema y a sus familiares, no como entes pasivos en el proceso, sino como protagonistas.

- De actividad formativa: dada la coyuntura que la Universidad Nacional y otras Universidades tengan los centros asistenciales citados como campos de formación para el profesional de ciencias de la salud (trabajadores sociales, enfermeras, sicólogos, terapistas, médicos), la clínica del dolor proporciona un contexto muy favorable para que el estudiante en formación se enfrente a problemas complejos adquiriendo experiencias que contribuirán a su desarrollo personal como profesional. Puede también aceptarse además que la clínica del dolor proporciona el foro en el cual la actividad de las diferentes disciplinas científicas, se evalúa en forma objetiva y no competitiva.

- De actividad investigativa: partiendo del conocimiento de nuestra realidad, generar también nuestro propio conocimiento y soluciones coherentes.

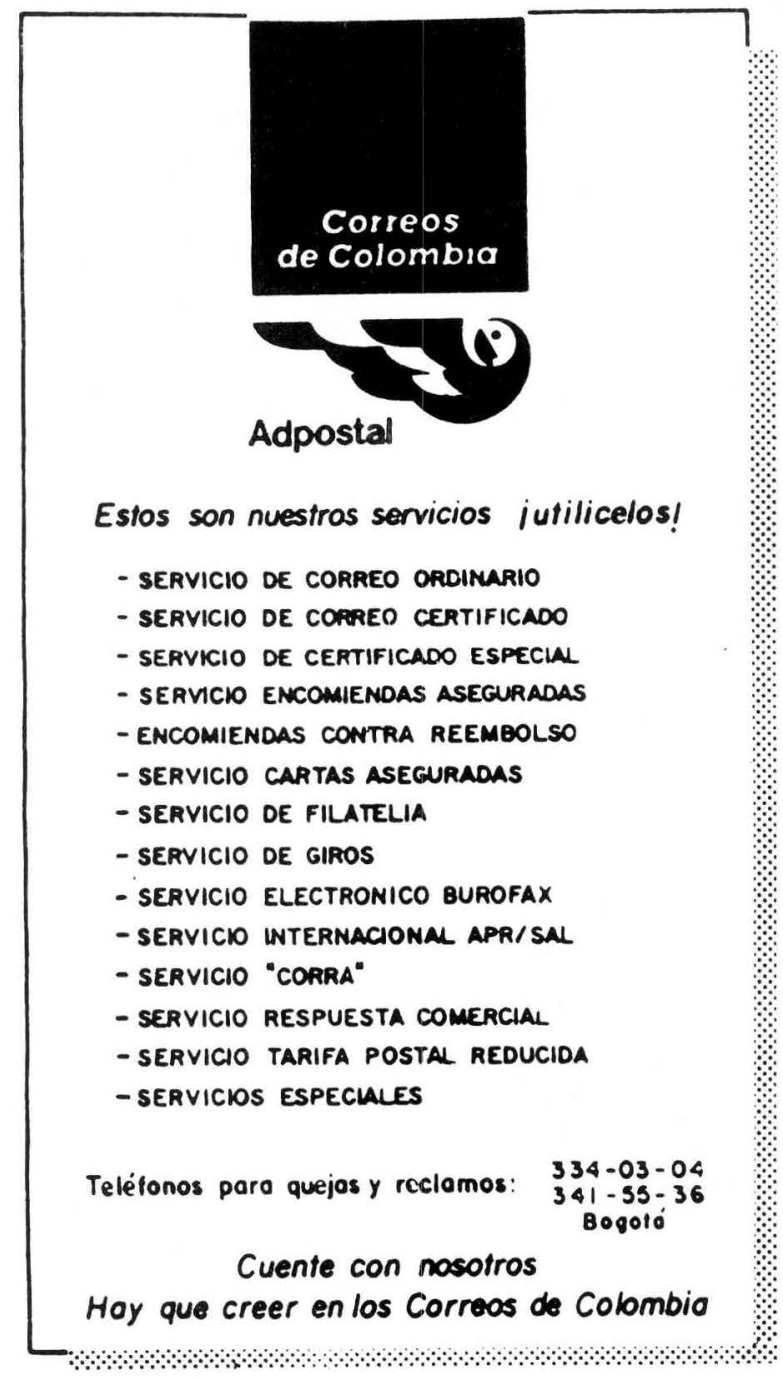

